\section{Outcomes for Indigenous and non-Indigenous patients who access treatment for hepatitis C in the Top End of the Northern Territory}

To THE EDITOR: Chronic hepatitis C virus (HCV) infection affects over 225000 Australians $^{1}$ and is a leading cause of the need for liver transplantation and of liver-related death, but curative treatments are available. Ethnicity is a major determinant of treatment responsiveness, with the lowest sustained virological response (SVR) rates reported in African patients, and the highest in Asian patients. ${ }^{2}$ Much of this difference is accounted for by racial differences in polymorphisms in the interleukin-28B (IL28B) gene; ${ }^{3}$ however, it is unknown how common these polymorphisms are in Indigenous Australians, and no studies have been published about hepatitis $C$ treatment outcomes among Indigenous Australians.

The hepatitis $C$ treatment service for the Top End of the Northern Territory is run from a community-based sexual health clinic in Darwin. As clinicians working at this service, our perception was that Indigenous people rarely accessed the service or received treatment for HCV infection. Further, we were concerned that - due to social, cultural and linguistic barriers - Indigenous people who accessed the service may be less likely to commence treatment and to successfully complete treatment and achieve an SVR. Following ethics approval from the Human Research Ethics Committee of the Northern Territory Department of Health and Families, we performed a retrospective case-note audit to determine the number of Indigenous people accessing the hepatitis $C$ treatment service and their characteristics and treatment outcomes.

During the period 1 January 2006 to 31 December 2010, 243 patients were seen on at least two occasions for assessment of HCV infection; all were adults and 22 (9\%) were Indigenous. During the audit period, HCV infection was treated with pegylated interferon- $\alpha$ plus ribavirin for $24-48$ weeks. There were no significant differences in the proportion of patients who went on to commence and complete treatment, and to achieve an SVR, between Indigenous and non-Indigenous patients (Box). Of five Indigenous patients tested for IL28B genotype, all had the favourable CC polymorphism at the rs12979860 locus. Compared with the unfavourable TT and CT

polymorphisms, the CC polymorphism at this locus is associated with at least a twofold higher chance of achieving a cure of HCV with interferon treatment, due to enhanced host immune responsiveness to interferon. ${ }^{3}$ the NT who access hepatitis C treatment services have a similar chance of achieving a cure (SVR) to nonIndigenous people. This may be partly because they are likely to carry the favourable CC polymorphism at the IL28B gene.
In conclusion, Indigenous people in

Indigenous
people in the NT
who access
hepatitis C

treatment services have a similar chance of achieving a cure ... to non-Indigenous people

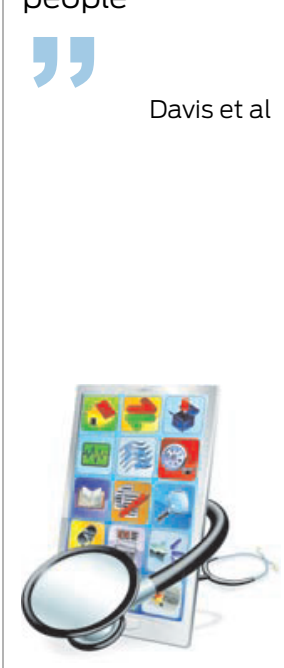

Indigenous compared with non-Indigenous patients who attended a hepatitis $C$ treatment service on at least two occasions for assessment of HCV infection*

\begin{tabular}{lccc} 
& Indigenous $(\boldsymbol{n}=22)$ & Non-Indigenous $(\boldsymbol{n}=221)$ & $P$ \\
\hline Age, median (interquartile range) & $41.0(36.2-45.0)$ & $47.3(39.3-52.1)$ & 0.14 \\
Men & $15 / 22(68 \%[45 \%-86 \%])$ & $144 / 221(65 \%[58 \%-71 \%])$ & 0.78 \\
HCV genotype 1 & $10 / 18(56 \%[31 \%-78 \%])$ & $87 / 173(50 \%[43 \%-58 \%])$ & 0.67 \\
Commenced HCV treatment & $11 / 22(50 \%[28 \%-72 \%])$ & $99 / 221(45 \%[38 \%-52 \%])$ & 0.58 \\
Completed HCV treatment & $9 / 11(82 \%[48 \%-98 \%])$ & $80 / 99(81 \%[72 \%-88 \%])$ & 0.94 \\
Achieved sustained virological response $^{\dagger}$ & $4 / 8(50 \%[16 \%-84 \%])$ & $54 / 88(61 \%[50 \%-72 \%])$ & 0.53 \\
\hline
\end{tabular}

$\mathrm{HCV}=$ hepatitis $\mathrm{C}$ virus. $*$ Data are number/denominator $(\%[95 \% \mathrm{Cl}])$ unless otherwise indicated. $\dagger$ Denominators represent those patients for whom 6-month post-treatment blood test results were available.
Joshua S Davis Senior Research Fellow? Anuja C Kulatunga General Practitioner ${ }^{2}$ Krispin Hajkowicz Infectious Diseases Physician ${ }^{3}$ 1 Global and Tropical Health, Menzies School of Health Research, Darwin, NT.

2 Karama Medical Centre, Darwin, NT. 3 Royal Brisbane and Women's Hospital, Brisbane, QLD. joshua.davis@menzies.edu.au

Competing interests: No relevant disclosures. doi: 10.5694/mjal3.10083

1 The Kirby Institute. HIV, viral hepatitis and sexually transmissible infections in Australia. Annual Surveillance Report 2012. Sydney: The Kirby Institute, the University of New South Wales, 2012

2 Muir AJ, Hu KQ, Gordon SC, et al. Hepatitis C treatment among racial and ethnic groups in the IDEAL trial. J Viral Hepat 2011; 18: el34-el43.

3 Ge D, Fellay J, Thompson AJ, et al. Genetic variation in IL28B predicts hepatitis C treatmentinduced viral clearance. Nature 2009; 461: 399-401. 